\title{
Fuzzy vector metric spaces and some results
}

\author{
Şehla Eminoğlu, Cüneyt Çevik* \\ Department of Mathematics, Faculty of Science, Gazi University, 06500 Teknikokullar Ankara, Turkey. \\ Communicated by C. Vetro
}

\begin{abstract}
The aim of this paper is to enrich the theory of fuzzy metric spaces through vectors. Additionally we define the concept of fuzzy vector diameter to be able to prove Cantor's intersection theorem and Baire's theorem in a different way. (c)2017 All rights reserved.
\end{abstract}

Keywords: Vector metric space, fuzzy vector metric space, Riesz space, fuzzy diameter. 2010 MSC: 54A40, 54E35, 06F20, 46A40.

\section{Introduction and preliminaries}

The theory of Zadeh's fuzzy sets [14] continues to be applied in many areas of mathematics. One of these is the study of Kramosil and Michálek, in which the concept of fuzziness is applied to the classical notions of metric spaces [7]. Later, George and Veeramani defined it again by making some modifications and gave some new results $[5,6]$. They associated the distance between two points of a set with a single non-negative real number, which is denoted by $t$. On the other hand, the notions of vector metric spaces in which the metric takes values of a Riesz space was firstly defined in [4]. Then, continuous functions between any two vector metric spaces were studied [3]. Our aim in this paper is to enrich the concept of fuzzy metric that is in the sense of George and Veeramani. Instead of the number $t$ we take in this paper any element of a Riesz space [2,8], namely any vector. In the space of continuous functions which is a Riesz space if we take the vector $t$ as a constant function, in this case every fuzzy metric is a fuzzy vector metric. To support our study in non-stationary fuzzy metric examples we consider normed Riesz spaces. Moreover, through lattice norm condition we can switch from order convergence in domain of the membership function to convergence in its range. Thus, we updated the continuity for the relevant fuzzy metric condition. Towards the end of the paper we define the concept of fuzzy vector diameter based on $[11,12]$ to give proof of some theorems in a different way from $[5,6]$. These theorems are located in functional analysis and topological spaces $[1,9,10,13]$ such as Cantor's intersection theorem and Baire's theorem.

Firstly we present basic concepts and results of $[2,8]$.

\footnotetext{
*Corresponding author

Email addresses: sehla_eminoglu@hotmail.com (Şehla Eminoğlu), ccevik@gazi.edu.tr (Cüneyt Çevik)
} 
Definition 1.1. Let $E$ be a real vector space. If $E$ has an order relation $\leqslant$ (i.e., $\leqslant$ is a reflexive, antisymmetric and transitive binary relation on $E$ ) which is compatible with the algebraic structure of $E$ in the sense that it satisfies the following conditions:

(1) if $s \leqslant t$, then $s+v \leqslant t+v$ holds for all $v \in E$;

(2) if $s \leqslant t$, then $\alpha s \leqslant \alpha t$ holds for all $\alpha \in \mathbb{R}^{+}$,

then $E$ is called ordered vector space.

For any two vectors $s$ and $t$ in $E$ we write $s<t$ to indicate $s \leqslant t$ but $s \neq t$. The notation $s \geqslant t$ is $t \leqslant s$ and the other notation $s>t$ is $t<s$. A vector $t$ in ordered vector space $E$ is called positive if $t>\theta$ holds. We denote the set of all positive vectors of $E$ with $E^{+}$.

An ordered vector space $E$ is called Riesz space if $E$ has the supremum and the infimum of the set $\{s, t\}$ for $s, t \in E$. We show the classical notation as follows:

$$
s \vee t=\sup \{s, t\} \text { and } s \wedge t=\inf \{s, t\}
$$

The absolute value of any vector $t$ in a Riesz space $E$ is defined by $|t|=t \vee(-t)$.

Function spaces are the important examples of Riesz spaces. A function space is an ordered vector space $E$ of real-valued functions on a set $\Omega$ with the pointwise ordering, that is $f \leqslant g$ holds in $E$ if and only if $f(x) \leqslant g(x)$ for all $x \in \Omega$. The lattice operations in any function spaces $E$ for each pair $f, g \in E$ is denoted by

$$
(f \vee g)(x)=\max \{f(x), g(x)\} \text { and }(f \wedge g)(x)=\min \{f(x), g(x)\} .
$$

A sequence $\left(t_{n}\right)$ in a Riesz space is called decreasing, which is denoted by $t_{n} \downarrow$ if $n \geqslant m$ implies $t_{n} \leqslant t_{m}$. The symbol $t_{n} \downarrow t$ means that $t_{n} \downarrow$ and $\inf \left\{t_{n}\right\}=t$ both hold. The meanings of $t_{n} \uparrow$ and $t_{n} \uparrow t$ are similar.

Definition 1.2. Let $E$ be a Riesz space. A sequence $\left(t_{n}\right)$ in $E$ is order convergent to some vector $t$, denoted $t_{n} \stackrel{o}{\rightarrow} t$, if there exists another sequence $\left(s_{n}\right)$ satisfying $\left|t_{n}-t\right| \leqslant s_{n} \downarrow \theta$.

Definition 1.3. Let $E$ be a Riesz space. A norm $\|\cdot\|$ on $E$ is called lattice norm if $|t| \leqslant|s|$ implies $\|t\| \leqslant\|s\|$. A Riesz space with lattice norm is said to be a normed Riesz space.

Vector metric spaces are first introduced in [4]. The values of the distance function are taken in a Riesz space.

Definition 1.4. Let $X$ be a non-empty set and $E$ be a Riesz space. The function $d: X \times X \rightarrow E$ is called vector metric (or E-metric) if it satisfies the following conditions:

(vm1) $\theta \leqslant d(x, y)$

(vm2) $d(x, y)=\theta$ if and only if $x=y$;

(vm3) $d(x, y)=d(y, x)$;

$(\operatorname{vm} 4) d(x, z) \leqslant d(x, y)+d(y, z)$

for all $x, y, z \in X$. Then $(X, d)$ is called vector metric space.

Because the set of real numbers $\mathbb{R}$ with the usual order $\leqslant$ is a Riesz space, every metric space is a vector metric space. Let $E$ be a Riesz space. The function $d: E \times E \rightarrow E$ defined as $d(x, y)=|x-y|$ is a vector metric. Hence, every Riesz space is a vector metric space. 


\section{Fuzzy vector metric spaces}

Definition 2.1 ([11]). A binary operation $*:[0,1] \times[0,1] \rightarrow[0,1]$ is a continuous t-norm if $*$ satisfies the following conditions:

(ct1) $*$ is associative and commutative;

(ct2) $*$ is continuous;

(ct3) $a * 1=a$, for all $a \in[0,1]$;

(ct4) $a * b \leqslant c * d$ whenever $a \leqslant c$ and $b \leqslant d$,

for all $a, b, c, d \in[0,1]$.

To establish the concept of fuzzy vector metric space we utilize the definition of fuzzy metric space that is in the sense of George and Veeramani.

Definition $2.2([5])$. Let $X$ be a non-empty set and $*$ be a continuous t-norm. The triple $(X, M, *)$ is called a fuzzy metric space if the fuzzy set $M$ on $X \times X \times(0, \infty)$ satisfies the following conditions:

(fm1) $M(x, y, t)>0$;

(fm2) $M(x, y, t)=1$ if and only if $x=y$;

(fm3) $M(x, y, t)=M(y, x, t)$;

(fm4) $M(x, y, t) * M(y, z, s) \leqslant M(x, z, t+s) ;$

$(\mathrm{fm} 5) \mathrm{M}(x, y,):.(0, \infty) \rightarrow[0,1]$ is continuous,

for all $x, y, z \in X$ and all $t, s>0$. If $(X, M, *)$ is a fuzzy metric space, we will say that $(M, *)$ or simply $M$ is a fuzzy metric on $X$.

Let us give the continuity for functionals defined on a Riesz space.

Definition 2.3. Let $E$ be a Riesz space a map $\varphi$ from $E$ to $\mathbb{R}$ is called continuous if for any sequence $\left(t_{n}\right)$ and $\mathrm{t} \in \mathrm{E}, \mathrm{t}_{\mathrm{n}} \stackrel{\mathrm{o}}{\rightarrow} \mathrm{t}$ implies $\varphi\left(\mathrm{t}_{\mathrm{n}}\right) \rightarrow \varphi(\mathrm{t})$ in $\mathbb{R}$.

We can now offer the fuzzy vector metric space concept based on the above definition.

Definition 2.4. Let $X$ be a non-empty set, $E$ be a Riesz space and $*$ be a continuous t-norm. The triple $\left(X, M^{E}, *\right)$ is called a fuzzy vector metric space if the fuzzy set $M^{E}$ on $X \times X \times E^{+}$satisfies the following conditions:

(fvm1) $M^{E}(x, y, t)>0$;

(fvm2) $M^{\mathrm{E}}(x, y, t)=1$ if and only if $x=y$;

(fvm3) $M^{E}(x, y, t)=M^{E}(y, x, t)$;

(fvm4) $M^{\mathrm{E}}(x, y, t) * M^{\mathrm{E}}(y, z, s) \leqslant M^{\mathrm{E}}(x, z, t+s)$;

(fvm5) $M^{E}(x, y,):. E^{+} \rightarrow[0,1]$ is continuous,

for all $x, y, z \in X$ and all $t, s \in E^{+}$. If $\left(X, M^{E}, *\right)$ is a fuzzy vector metric space, we will say that $\left(M^{E}, *\right)$ or simply $M^{\mathrm{E}}$ is a fuzzy vector metric on $\mathrm{X}$.

It is obvious that both fuzzy metric spaces and vector metric spaces are fuzzy vector metric spaces. 
Lemma 2.5. $\mathrm{M}^{\mathrm{E}}(\mathrm{x}, \mathrm{y},):. \mathrm{E}^{+} \rightarrow[0,1]$ is nondecreasing for all $\mathrm{x}, \mathrm{y} \in \mathrm{X}$.

Example 2.6. Let $X$ be a non-empty set and $E$ be a normed Riesz space and $d: X \times X \rightarrow E$ be a vector metric on $E$. Define $a * b=\min \{a, b\}$ and

$$
M^{E}(x, y, t)=\frac{\|t\|}{\|t\|+\|d(x, y)\|},
$$

for all $x, y \in X$ and $t \in E^{+}$. Then $\left(X, M^{E}, *\right)$ is a fuzzy vector metric space.

Proof. We only show the fifth condition. Let $\left(t_{n}\right)$ be an order convergent sequence to $t$. Then, there exists another sequence $\left(s_{n}\right)$ satisfying $\left|t_{n}-t\right| \leqslant s_{n} \downarrow 0$. From the lattice norm condition and the inverse triangle inequality of norm we obtain

$$
\left\|t_{n}\right\|-\|t\| \mid \leqslant\left\|t_{n}-t\right\| \leqslant\left\|s_{n}\right\| \downarrow 0,
$$

for all $n \in \mathbb{N}^{+}$. So we get

$$
\lim _{n \rightarrow \infty} M^{E}\left(x, y, t_{n}\right)=\frac{\lim _{n \rightarrow \infty}\left\|t_{n}\right\|}{\lim _{n \rightarrow \infty}\left\|t_{n}\right\|+\|d(x, y)\|}=\frac{\|t\|}{\|t\|+\|d(x, y)\|}=M^{E}(x, y, t) .
$$

Example 2.7. Let $X=\mathbb{R} \backslash\{0\}$ and $E$ be a Riesz space. Define $a * b=a . b$ and

$$
M^{E}(x, y, t)= \begin{cases}|x| /|y|, & |x| \leqslant|y| \\ |y| /|x|, & |y| \leqslant|x|\end{cases}
$$

for all $x, y \in X$. Then $\left(X, M^{E}, *\right)$ is a fuzzy vector metric space.

Example 2.8. Let $X$ be a non-empty set, $E$ be a normed Riesz space and $\varphi:(0, \infty) \rightarrow[0,1]$ an increasing continuous function. Define $a * b=a . b$ and

$$
M^{E}(x, y, t)= \begin{cases}1, & x=y \\ \varphi(\|t\|), & x \neq y\end{cases}
$$

for all $x, y \in X$ and $t \in E^{+}$. Then $\left(X, M^{E}, *\right)$ is a fuzzy vector metric space.

Example 2.9. Let $X$ be a normed Riesz space and $E$ be a Riesz space. Define $a * b=a . b$ and

$$
M^{E}(x, y, t)= \begin{cases}1, & x=y \\ \|x \vee y\| /\||x| \vee|y|\|, & x \neq y\end{cases}
$$

for all $x, y \in X$ and $t \in E^{+}$. Then $\left(X, M^{E}, *\right)$ is a fuzzy vector metric space.

Now let us introduce some topological concepts for fuzzy vector metric spaces.

Definition 2.10. Let $\left(X, M^{E}, *\right)$ be a fuzzy vector metric space. We define open ball $B^{E}(x, r, t)$ and closed ball $\mathrm{B}^{\mathrm{E}}[x, r, t]$ for $t \in \mathrm{E}^{+}$with centre $x \in X$ and radius $r, 0<r<1$ as

$$
\begin{aligned}
& B^{E}(x, r, t)=\left\{y \in X: M^{E}(x, y, t)>1-r\right\}, \\
& B^{E}[x, r, t]=\left\{y \in X: M^{E}(x, y, t) \geqslant 1-r\right\},
\end{aligned}
$$

respectively. 


\section{Definition 2.11.}

(a) Let $\left(X, M^{E}, *\right)$ be a fuzzy vector metric space and $A \subseteq X$. Then $A$ is called $F_{E}$-bounded if for every $x, y \in A$ and $t \in E^{+}$there exists an $r \in(0,1)$ such that $M^{E}(x, y, t) \geqslant 1-r$. Furthermore, a sequence $\left(x_{n}\right)$ in $X$ is said to be $F_{E}$-bounded if for all $t \in E^{+}$and for all $n \in \mathbb{N}^{+}$there exists an $r \in(0,1)$ such that $\left(x_{n}\right) \subseteq B^{E}[x, r, t]$.

(b) A sequence $\left(x_{n}\right)$ in fuzzy vector metric space $\left(X, M^{E}, *\right)$ converges to $x \in X$ if for each $\varepsilon \in(0,1)$ and $t \in E^{+}$there exists $n_{0}=n_{0}(\varepsilon) \in \mathbb{N}$ such that $M^{E}\left(x_{n}, x, t\right)>1-\varepsilon$ for all $n \geqslant n_{0}$ and it is denoted by

$$
\lim _{n \rightarrow \infty} M^{E}\left(x_{n}, x, t\right)=1 \text { or } x_{n} \stackrel{M^{E}}{\rightarrow} x
$$

(c) A sequence $\left(x_{n}\right)$ in a fuzzy vector metric space $\left(X, M^{E}, *\right)$ is called Cauchy if for each $\varepsilon \in(0,1)$ and $t \in E^{+}$there exists $n_{0} \in \mathbb{N}$ such that $M^{E}\left(x_{n}, x_{m}, t\right)>1-\varepsilon$ for all $n, m \geqslant n_{0}$.

(d) A fuzzy vector metric space $\left(X, M^{E}, *\right)$ is called complete if every Cauchy sequence in $X$ converges.

(e) A subset $Y$ of a fuzzy vector metric space $\left(X, M^{E}, *\right)$ is called closed if $\left(x_{n}\right) \subseteq Y$ and $x_{n} \stackrel{M^{E}}{\rightarrow} x$ imply $x \in \mathrm{Y}$.

Theorem 2.12. Let $\left(X, M^{\mathrm{E}}, *\right)$ be a fuzzy vector metric space and $\left(x_{\mathrm{n}}\right)$ be a convergent sequence in $\mathrm{X}$. Then

(i) $\left(x_{n}\right)$ is $\mathrm{F}^{\mathrm{E}}$-bounded and its limit is unique;

(ii) $\left(x_{n}\right)$ is a Cauchy sequence in $X$;

(iii) every subsequence of $\left(x_{n}\right)$ converges to the same limit.

Proof.

(i) First, we show that the convergent sequence $\left(x_{n}\right)$ is $\mathrm{F}^{\mathrm{E}}$-bounded. Suppose that $x_{n} \stackrel{M^{E}}{\rightarrow} x$. Then, for each $\varepsilon \in(0,1)$ and $t \in E^{+}$, there exists $n_{0} \in \mathbb{N}$ such that $M^{E}\left(x_{n}, x, t / 2\right)>1-\varepsilon$ for all $n \geqslant n_{0}$. Now, for $x_{0} \in X$ and $s \in(0,1)$, let $M^{\mathrm{E}}\left(x_{0}, x, t / 2\right)>1-s$ and, for $k \in(0,1)$, let

$$
\min \left\{M^{E}\left(x_{n}, x, t / 2\right): n=1,2, \cdots, n_{0}\right\}=1-k .
$$

Then we can find a number $r \in(0,1)$ such that

$$
\min \{(1-\mathrm{s}) *(1-\mathrm{k}),(1-\mathrm{s}) *(1-\varepsilon)\}=1-\mathrm{r} .
$$

So, for all $n \in \mathbb{N}^{+}$we have

$$
M^{E}\left(x_{0}, x_{n}, t\right) \geqslant M^{E}\left(x_{0}, x, t / 2\right) * M^{E}\left(x_{n}, x, t / 2\right) \geqslant 1-r .
$$

Thus, for all $n \in \mathbb{N}^{+},\left(x_{n}\right) \subseteq B^{E}\left[x_{0}, r, t\right]$, that is, $\left(x_{n}\right)$ is $F_{E}$-bounded.

Now, we show that the limit of $\left(x_{n}\right)$ is unique. We assume that the convergent sequence $\left(x_{n}\right)$ has two different limits $x$ and $y$. For any $t \in E^{+}$and any $\varepsilon \in(0,1)$, we can find a number $r \in(0,1)$ such that $(1-r) *(1-r) \geqslant 1-\varepsilon$. Let $\varepsilon=1-M^{E}(x, y, t)$. According to our assumption there exists $n_{1} \in \mathbb{N}$ such that $M^{E}\left(x_{n}, x, t / 2\right)>1-r$, for all $n \geqslant n_{1}$ and there exists $n_{2} \in \mathbb{N}$ such that $M^{E}\left(x_{n}, y, t / 2\right)>1-r$, for all $n \geqslant n_{2}$. When we take $n_{0}=\max \left\{n_{1}, n_{2}\right\}$, then for $n \geqslant n_{0}$ we have

$$
M^{E}(x, y, t) \geqslant M^{E}\left(x_{n}, x, t / 2\right) * M^{E}\left(x_{n}, y, t / 2\right)>(1-r) *(1-r) \geqslant 1-\varepsilon=M^{E}(x, y, t),
$$

that is, $M^{E}(x, y, t)>M^{E}(x, y, t)$, which is not possible. 
(ii) Let $t \in \mathrm{E}^{+}$and $\varepsilon \in(0,1)$. We can find a number $r \in(0,1)$ such that $(1-r) *(1-r)>(1-\varepsilon)$. Since $\left(x_{n}\right)$ is a convergent sequence, there exists $n_{0} \in \mathbb{N}$ such that $M^{E}\left(x_{n}, x, t / 2\right)>1-r$, for all $n \geqslant n_{0}$. For all $n, m \geqslant n_{0}$ we get

$$
M^{E}\left(x_{n}, x_{m}, t\right) \geqslant M^{E}\left(x_{n}, x, t / 2\right) * M^{E}\left(x, x_{m}, t / 2\right) \geqslant(1-r) *(1-r)>(1-\varepsilon) .
$$

(iii) Let $\left(x_{n_{k}}\right)$ be a subsequence of $\left(x_{n}\right)$. If $x_{n} \stackrel{M^{E}}{\rightarrow} x$, then for each $\varepsilon \in(0,1)$ and $t \in E^{+}$, there exists $n_{0} \in \mathbb{N}$ such that $M^{E}\left(x_{n}, x, t / 2\right)>1-\varepsilon$, for all $n \geqslant n_{0}$. If $k \geqslant n_{0}$, then $n_{0} \leqslant k \leqslant n_{k}$ and hence $M^{E}\left(x_{n_{k}}, x, t\right)>1-\varepsilon$.

Proposition 2.13. Suppose that $\left(\mathrm{X}_{1}, \mathrm{M}_{1}^{\mathrm{E}}, *\right)$ and $\left(\mathrm{X}_{2}, \mathrm{M}_{2}^{\mathrm{E}}, *\right)$ are two fuzzy vector metric spaces. The fuzzy set $\mathrm{M}^{\mathrm{E}}$ defined as

$$
M^{E}\left(\left(x_{1}, x_{2}\right),\left(y_{1}, y_{2}\right), t\right)=M_{1}^{E}\left(x_{1}, y_{1}, t\right) * M_{2}^{E}\left(x_{2}, y_{2}, t\right),
$$

for $\left(\mathrm{x}_{1}, \mathrm{x}_{2}\right),\left(\mathrm{y}_{1}, \mathrm{y}_{2}\right) \in \mathrm{X}_{1} \times \mathrm{X}_{2}, \mathrm{t} \in \mathrm{E}^{+}$is a fuzzy vector metric on $\mathrm{X}_{1} \times \mathrm{X}_{2}$.

Therefore, we can give the following result.

Theorem 2.14. Let $\left(\mathrm{X}_{1}, \mathrm{M}_{1}^{\mathrm{E}}, *\right)$ and $\left(\mathrm{X}_{2}, \mathrm{M}_{2}^{\mathrm{E}}, *\right)$ be two fuzzy vector metric spaces and $\mathrm{M}^{\mathrm{E}}$ be the fuzzy vector metric as in Proposition 2.13. For any two sequences $\left(x_{n}\right)$ and $\left(y_{n}\right)$, if $x_{n} \stackrel{M_{1}^{E}}{\rightarrow} x$ in $X_{1}$ and $y_{n} \stackrel{M_{2}^{E}}{\rightarrow} y$ in $X_{2}$, then $\left(x_{n}, y_{n}\right) \stackrel{M^{E}}{\rightarrow}(x, y)$ in $X_{1} \times X_{2}$.

Proof. Let $\mathrm{t} \in \mathrm{E}^{+}$and $\varepsilon \in(0,1)$. We can find a number $r \in(0,1)$ such that $(1-r) *(1-r)>(1-\varepsilon)$. Then, there exists $n_{1} \in \mathbb{N}$ such that $M_{1}^{E}\left(x_{n}, x, t\right)>1-r$, for all $n \geqslant n_{1}$ and there exists $n_{2} \in \mathbb{N}$ such that $M_{2}^{E}\left(y_{n}, y, t\right)>1-r$, for all $n \geqslant n_{2}$. Choosing $n_{0}=\max \left\{n_{1}, n_{2}\right\}$, we have

$$
M^{E}\left(\left(x_{n}, y_{n}\right),(x, y), t\right)=M_{1}^{E}\left(x_{n}, x, t\right) * M_{2}^{E}\left(y_{n}, y, t\right)>(1-r) *(1-r)>(1-\varepsilon) .
$$

This completes the proof of theorem.

Theorem 2.15. For the fuzzy vector metric space $\left(X, M^{\mathrm{E}}, *\right)$ the followings hold:

(i) every convergent sequence is a Cauchy sequence;

(ii) every Cauchy sequence is $\mathrm{F}^{\mathrm{E}}$-bounded;

(iii) if a Cauchy sequence $\left(x_{n}\right)$ in $X$ has a subsequence $\left(x_{n_{k}}\right)$ such that $x_{n_{k}} \stackrel{M^{E}}{\rightarrow} x$, then $x_{n} \stackrel{M^{E}}{\rightarrow} x$.

Proof.

(i) Let $t \in E^{+}$and $\varepsilon \in(0,1)$. We can find a number $r \in(0,1)$ such that $(1-r) *(1-r)>1-\varepsilon$. If $x_{n} \stackrel{M^{E}}{\rightarrow} x$, then there exists $n_{0} \in \mathbb{N}$ such that $M^{E}\left(x_{n}, x, t / 2\right)>1-r$, for all $n \geqslant n_{0}$. Hence for $n, m \geqslant n_{0}$, we have

$$
M^{E}\left(x_{n}, x_{m}, t\right) \geqslant M^{E}\left(x_{n}, x, t / 2\right) * M^{E}\left(x_{m}, x, t / 2\right)>(1-r) *(1-r)>1-\varepsilon .
$$

(ii) Let $\left(x_{n}\right)$ be a Cauchy sequence in $X$. Then, for each $\varepsilon \in(0,1)$ and $t \in E^{+}$, there exists $n_{0} \in \mathbb{N}$ such that $M^{E}\left(x_{n}, x_{m}, t\right)>1-\varepsilon$, for all $n, m \geqslant n_{0}$. So, for $n \geqslant n_{0}$ we have $M^{E}\left(x_{n}, x_{n_{0}}, t\right)>1-\varepsilon$. Let

$$
r=1-\min \left\{M^{E}\left(x_{n}, x_{n_{0}}, t\right): n=1,2, \cdots, n_{0}-1\right\},
$$

and choose $s=\max \{\varepsilon, r\}$. Then $\left\{x_{n}: n=1,2, \cdots\right\} \subseteq \bar{B}\left(x_{n_{0}}, s, t\right)$, that is, $\left(x_{n}\right)$ is $F_{E}$-bounded.

(iii) Let $t \in E^{+}$and $\varepsilon \in(0,1)$. We can find $r \in(0,1)$ such that $(1-r) *(1-r) \geqslant 1-\varepsilon$. Since $\left(x_{n}\right)$ is a Cauchy sequence in $X$, there exists $n_{0} \in \mathbb{N}$ such that $M^{E}\left(x_{n}, x_{m}, t / 2\right)>1-r$, for all $m, n \geqslant n_{0}$. From $x_{n_{k}} \stackrel{M^{E}}{\rightarrow} x$ there exists positive integer $i_{k}$ such that $i_{k}>n_{0}$ and $M^{E}\left(x_{i_{k}}, x, t / 2\right)>1-r$. For every $n \geqslant n_{0}$, we have

$$
M^{E}\left(x_{n}, x, t\right) \geqslant M^{E}\left(x_{n}, x_{i_{p}}, t / 2\right) * M^{E}\left(x_{i_{p}}, x, t / 2\right)>(1-r) *(1-r) \geqslant 1-\varepsilon,
$$

and so, $x_{n} \stackrel{M^{E}}{\rightarrow} x$. 
Definition 2.16. Let $\left(X, M^{E}, *\right)$ be a fuzzy vector metric space and $A$ be a non-empty subset of $X$. We define fuzzy vector diameter $\mathrm{D}_{\AA}^{\mathrm{E}}$ as

$$
D_{\AA}^{E}=\sup _{t \in E^{+}}\left\{\inf M^{E}(x, y, t): x, y \in A\right\} .
$$

Theorem 2.17. The fuzzy vector diameter $\mathrm{D}_{\mathrm{A}}^{\mathrm{E}}$ has the following properties.

(i) $\mathrm{D}_{\mathrm{A}}^{\mathrm{E}}=1$ if and only if $\mathrm{A}$ is a singleton set.

(ii) If $\mathrm{A} \subseteq \mathrm{B}$, then $\mathrm{D}_{\mathrm{A}}^{\mathrm{E}} \geqslant \mathrm{D}_{\mathrm{B}}^{\mathrm{E}}$.

(iii) For any $x, y \in A, M^{E}(x, y, t) \geqslant D_{A}^{E}$ holds.

(iv) If $A=\{x, y\}$, then $D_{A}^{E}=M^{E}(x, y, t)$.

(v) If $\mathrm{A} \cap \mathrm{B}$ is nonempty set, then $\mathrm{D}_{\mathrm{A} \cup \mathrm{B}}^{\mathrm{E}} \geqslant \mathrm{D}_{\mathrm{A}}^{\mathrm{E}} * \mathrm{D}_{\mathrm{B}}^{\mathrm{E}}$.

Definition 2.18. Let $\left(X, M^{E}, *\right)$ be a fuzzy vector metric space and $\left(A_{n}\right)$ be a sequence of non-empty subsets in $X$. We call the sequence $\left(A_{n}\right)$ has appearing fuzzy vector diameter if

$$
\lim _{n \rightarrow \infty} D_{\AA_{n}}^{E}=1 \text {. }
$$

Then, for each $r \in(0,1)$ and $t \in E^{+}$, there exists $n_{0} \in \mathbb{N}$ such that $M^{E}(x, y, t)>1-r$, for all $x, y \in A_{n_{0}}$.

Theorem 2.19 (Cantor's intersection theorem). Let $\left(X, M^{\mathrm{E}}, *\right)$ be a fuzzy vector metric space and $\left(A_{n}\right)$ be any decreasing sequence of nonempty closed subsets of $\mathrm{X}$ with appearing fuzzy vector diameter. $\mathrm{X}$ is complete if and only if there is exactly one point $x \in X$ such that $\bigcap_{n=1}^{\infty} A_{n}=\{x\}$.

Proof. Let the fuzzy vector metric space $X$ be complete. We can form a sequence $\left(x_{n}\right)$ by taking a point $x_{n} \in A_{n}$ for each $n \in \mathbb{N}$. If we choose $m \geqslant n$, we get $A_{m} \subseteq A_{n}$, so that all points $\left\{x_{m}: m \geqslant n\right\}$ of the sequence belong to the set $A_{n}$. So, for each $t \in E^{+}$we obtain $M^{E}\left(x_{m}, x_{n}, t\right) \geqslant D_{A_{n}}^{E}$ for all $m \geqslant n$ and, consequently $\lim _{n, m \rightarrow \infty} M^{E}\left(x_{m}, x_{n}, t\right)=1$. In this case $\left(x_{n}\right)$ is a Cauchy sequence. Since $X$ is complete, there exists a point $x \in X$, such that $\lim _{n \rightarrow \infty} M^{E}\left(x_{n}, x, t\right)=1$. We take a set $A_{n_{0}}$. The limit of the sequence $\left\{x_{n}: n \geqslant n_{0}\right\} \subset A_{n_{0}}$ is of course the same point $x$. Furthermore, since $A_{n_{0}}$ is closed, $x \in A_{n_{0}}$. This means that $x$ belongs to every member of the sequence $\left(A_{n}\right)$. Hence we get $x \in \bigcap_{n=1}^{\infty} A_{n}$. Now we consider another point $x^{\prime} \in \bigcap_{n=1}^{\infty} A_{n}$. Then, for all $t \in E^{+}$the relation $M^{E}\left(x, x^{\prime}, t\right) \geqslant D_{A_{n}}^{E}$ must hold and, this implies that $M^{E}\left(x, x^{\prime}, t\right)=1$. This means that $\bigcap_{n=1}^{\infty} A_{n}=\{x\}$ because of $x=x^{\prime}$.

Conversely, let $\left(x_{n}\right)$ be a Cauchy sequence in $X$ and for $n \in \mathbb{N}, A_{n}=\overline{\left\{x_{m}: m \geqslant n\right\}}$ be a closed, nonempty subset of $X$. Since the sequence $\left(A_{n}\right)$ is decreasing and since $\left(x_{n}\right)$ is a Cauchy sequence, we must have $\lim _{n \rightarrow \infty} D_{A_{n}}^{E}=1$. To our assumption the intersection of all these sets contains only a single point of the space $X$. Let us assume that $\bigcap_{\mathfrak{n}=1}^{\infty} A_{\mathfrak{n}}=\{x\}$. For each $\varepsilon \in(0,1)$, there exists $n_{0} \in \mathbb{N}$ such that $D_{A_{n_{0}}}^{E}>1-\varepsilon$. We know that $x \in A_{n_{0}}$, thus for each $\varepsilon \in(0,1)$ and $t \in E^{+}$we get $M^{E}\left(x_{n}, x, t\right)>1-\varepsilon$ for all $n \geqslant n_{0}$. Therefore, the arbitrary Cauchy sequence $\left(x_{n}\right)$ converges to the point $x$. This means that the fuzzy vector metric space $X$ is complete.

Theorem 2.20 (Baire's theorem). Let $X$ be a complete fuzzy vector metric space and let $\left\{A_{n} \subset X: n=1,2, \cdots\right\}$ be a countable family of open subsets which are dense in $\mathrm{X}$. Then the set $\bigcap_{n=1}^{\infty} A_{n}$ is also dense in $\mathrm{X}$.

Proof. In order to prove the theorem, we have to show, for all $x \in X, 0<r<1$ and $t \in E^{+}$, the intersection of open ball $B^{E}(x, r, t)$ with the set $\bigcap_{n=1}^{\infty} A_{n}$ is not empty. First we take the set $A_{1}$. Since $A_{1}$ is dense in $X, B^{E}(x, r, t) \cap A_{1}$ is open and non-empty. Let $x_{1} \in B^{E}(x, r, t) \cap A_{1}$, then there exist $r_{1} \in(0,1)$ and $t_{1} \in E^{+}$ such that $B^{E}\left[x_{1}, r_{1}, t_{1}\right] \subset B^{E}(x, r, t) \cap A_{1}$. Let $B_{1}^{E}=B^{E}\left(x_{1}, r_{1}, t_{1}\right)$. Since $A_{2}$ is dense in $X, B_{1}^{E} \cap A_{2}$ is open and nonempty. Let $x_{2} \in B_{1}^{E} \cap A_{2}$, then there exist $r_{2} \in\left(0, \frac{1}{2}\right)$ and $t_{2} \in E^{+}$such that $B^{E}\left[x_{2}, r_{2}, t_{2}\right] \subset B_{1}^{E} \cap A_{2}$. 
Let $B_{2}^{E}=B^{E}\left(x_{2}, r_{2}, t_{2}\right)$. If we proceed inductively, we obtain a sequence $\left(x_{n}\right)$ in $X$ and a sequence $\left(r_{n}\right)$ of positive real numbers such that

$$
B^{E}\left[x_{n+1}, r_{n+1}, t_{n+1}\right] \subset B_{n}^{E} \cap A_{n+1} \subset B^{E}\left[x_{n}, r_{n}, t_{n}\right] \text { and } r_{n} \in\left(0, \frac{1}{n}\right)
$$

for all $n \in \mathbb{N}$. Theorem 2.19 guarantees that $\bigcap_{n=1}^{\infty} B^{E}\left[x_{n}, r_{n}, t_{n}\right]$ is a singleton. From

$$
\bigcap_{n=1}^{\infty} B^{E}\left[x_{n}, r_{n}, t_{n}\right] \subset B^{E}(x, r, t) \cap\left(\bigcap_{n=1}^{\infty} A_{n}\right),
$$

we obtain that $B^{E}(x, r, t) \cap\left(\bigcap_{n=1}^{\infty} A_{n}\right) \neq \varnothing$.

\section{References}

[1] C. D. Aliprantis, K. C. Border, Infinite-dimensional analysis, A hitchhiker's guide, Second edition, Springer-Verlag, Berlin, (1999). 1

[2] C. D. Aliprantis, O. Burkinshaw, Positive operators, Reprint of the 1985 original, Springer, Dordrecht, (2006). 1

[3] C. Çevik, On continuity of functions between vector metric spaces, J. Funct. Space, 2014 (2014), 6 pages. 1

[4] C. Çevik, I. Altun, Vector metric spaces and some properties, Topol. Methods Nonlinear Anal., 34 (2009), 375-382. 1, 1

[5] A. George, P. Veeramani, On some results in fuzzy metric spaces, Fuzzy Sets and Systems, 64 (1994), 395-399. 1, 2.2

[6] A. George, P. Veeramani, On some results of analysis for fuzzy metric spaces, Fuzzy Sets and Systems, 90 (1997), 365-368. 1

[7] I. Kramosil, J. Michálek, Fuzzy metrics and statistical metric spaces, Kybernetika (Prague), 11 (1975), 336-344. 1

[8] W. A. J. Luxemburg, A. C. Zaanen, Riesz spaces, Vol. I, North-Holland Mathematical Library, North-Holland Publishing Co., Amsterdam-London; American Elsevier Publishing Co., New York, (1971). 1

[9] J. R. Munkres, Topology: a first course, Prentice-Hall, Inc., Englewood Cliffs, N.J., (1975). 1

[10] W. Rudin, Functional analysis, Second edition, International Series in Pure and Applied Mathematics, McGrawHill, Inc., New York, (1991). 1

[11] B. Schweizer, A. Sklar, Statistical metric spaces, Pacific J. Math., 10 (1960), 313-334. 1, 2.1

[12] B. Schweizer, A. Sklar, Probabilistic metric spaces, North-Holland Series in Probability and Applied Mathematics, North-Holland Publishing Co., New York, (1983). 1

[13] E. S. Şuhubi, Functional analysis, Kluwer Academic Publishers, Dordrecht, (2003). 1

[14] L. A. Zadeh, Fuzzy sets, Information and Control, 8 (1965), 338-353. 1 